REYISTA

dete

CEPA

NUMERO 62

AGOSTO 1997

SANTIAGO DE CHILE

OSCAR ALTIMIR

Direcsor

EUGENIO LAHERA

Secretario Técnico

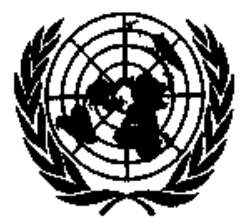

NACIONES UNIDAS 
Estado, comunidad y sociedad en el desarrollo social

Fermando Henrique Cardoso

Un balance de las reformas estructurales neoliberales en América Latina

Joseph Ramos

Deuda y sostenibilidad ffscal: ise repite la historia?

Guillermo E. Perry

Reformas petroleras: las opciones en juego

Fernando Sánchez Albavera

Las organizaciones indígenas: actores emergentes en

América Latina

Rodolfo Stavenhagen

El empleo rural no agropecuario en el Istmo Centroamericano

Jürgen Weller

Marginalidad e integración social en Uruguay

Rubén Kaztman

La política comercial en el marco de la Organización Mundial

de Comercio

Diana Tussie

Comercio y medio ambiente: ¿luz verde o luz roja?

Helga Hoffmann

Anclas nominales y escenarios de coordinación macroeconómica en el MERCoSUR

Gonzalo Rodríguez Prada

Políticas de promoción de exportaciones en Centroamérica

Larry Willmore

Publicaciones recientes de la CEPAL 


\section{Anclas nominales}

\section{y escenarios de coordinación macroeconómica en el MERCOSUR}

\section{Gonzalo Rodríguez Prada}

Universidad de Alcala, Espuña
En este trabajo se aborda el estudio de la coordinación macro económica en el entomo del Mercosur, analizando la contribución de diferentes anclas nominales (monetarias y cambiarias) al logro de los objetivos de convergencia nominal y crecimien. to económico sostenido. Se exploran las posibles ganancias de bienestar asociadas a la coordinación de las políticas, a fin de racionalizar las propuestas que abogan por una mayor cootdinación monetaria. Se estudia el problema de seleccionar el ancla nominal más adecuada para servir de base al acuerdo cooperativo, en una economía afectada por perturbaciones reales y monetarias. Tras considerar las diferencias estructurales existentes entre las economías que forman parte del Mercosur, se señala la necesidad de una coordinación macroeconómica flexible en el entomo de este bloque regional. En particular se examinan, en el contexto de un modelo centro-periferia de coordinación monetaria, Jos problemas de sostenibilidad de un mecanismo de coordinación monetaria de carácter asimétrico basado en la utilización del tipo de cambio como ancla nominal. En seguida se plantea una propuesta de coordinación macroeconómica sostenible en virtud de un acuerdo cambiario Mexible, sustentado a su vez en un mecanismo de bandas de fluctuación reforzado por criterios de convergencia nominal, reformas estructurales relativas a la flexibilización de los mercados y, eventualmente, controles moderados a los movimientos de capitales. El trabajo concluye con algunas reflexiones en torno al diseño de una estrategia para un abandono gradual en Argentina del mecanismo de cuasi caja de conversión, con miras a posibilitar la coordinación macroeconomica en el Mercosur. 
I

\section{Introducción}

La consolidación del Mercosur como un proyecto viable de integración económica está acentuando cada vez en mayor medida la necesidad de poner en marcha un programa bien articulado de coordinación macroeconómica entre los países que forman este bloque regional. ${ }^{1}$ La economía argentina, una de las más cerradas del mundo en el momento de la firma del Tratado de Asunción, fue la que experimentó el avance más significativo en el proceso de apertura comercial al exterior. Un rápido examen de las magnitudes del comercio exterior de este bloque regional muestra que las posibilifades del comercio intrarregional son todavía muy importantes. ${ }^{2} \mathrm{Si}$ bien los países más pequeños del Mercosur muestran una fuerte dependencia comercial de sus socios más grandes, Argentina y Brasil tienen una vocación declarada por el comercio global. ${ }^{3} \mathrm{La}$

[.] El autor agradece al Institute of Business and Economic Research (BBER) de la Universidad de California, Berkeley, las facilidades dadas para electuar esta investigación. Agradece también los útiles comentarios formulados por un evaluador anónimo de este trabajo. 'El dinamismo del Mercosur se pone de manifiesto al observar que el comercio entre los países de este bloque regional se duplicó en menos de cuatro aíos, tras la tírma del Tratado de Asunción en marzo de 1991. El comercio intrartegional del Mercosur pasó de 3639 millones de dolares en 1990 a casi 13000 millones de dólares en 1995. A partir de 1992 Argentina se convirtió en el segundo mercallo en importancia (después de Estados Unidos) para Brasil, absorbiendo casi el 9\% de las exportaciones brasilenas en 1995. A su vez, Brasil es el principal comprador de productos argentinos manufacturados y primarios de origen agrícota. En el período 19911995 este pás acumuló un superávit comercial de unos 4800 milones de dólares con el resto de sus socios del Mercosur, a pesar de los signifteativos deficit registrados con Argentina en 1991 y 1995.

${ }^{2}$ El grado de apertura de Argentina pasó de sólo $7 \%$ en 1991 a más de 17\% en 1995. A lo largo de este período el de Brasil se mantuvo estabilizado en torno al $15 \%$; el de Uruguay oscilo entre el 40 y el 45\%: y Paraguay continú siendo la economía más abierta del grupo, con un indice cercano al $80 \%$ como promedio. Aproximadamente el $30 \%$ del comercio total de los países del Mercosur se realiza con la Unión Europea, el $20 \%$ con Norteamérica y el $12 \%$ con el este de Asia. El comercio intrartegional todavía se sitúa por debajo del $20 \%$, mientras que aquél con el resto de los países latinoamericanos apenas alcanza el $10 \%$ del total.

${ }^{3}$ Mieniras que en el caso argentino esta vecación es nuy reciente y todavía no está plenamente consolidada, la política comercial brasiletha se ha guindo conscientemente por este principio desde hace varias décadas. En la actualidad Brasil concentra en la Unión Europen alrededor del $30 \%$ de sus exportaciones, mientras que Estado: Unidos absorbe otro 20 a $25 \%$ de eltas (10 mismo que A mérica Latina). Otro mercado importante es el del este de Asia, al que Brasil destina entre el 15 y el $20 \%$ de las exportaciones totales. Esa tradición puede haber favorecido la apuesta del Mercosur por un regionalismo abierto que parece vislumbrarse en la actualidad. En el caso de Uruguay casi el $40 \%$ de sus exportaciones y el $50 \%$ de sus importaciones se realizan con el Mercosur; Paraguay destina al Mercosur el $45 \%$ de sus exportaciones y obtiene de sus socios el $40 \%$ de sus importaciones. actual estrategia de apertura externa, que parece servir a los intereses de todas las partes del acuerdo comercial, tiene dos pilares fundamentales. En primer lugar, la ampliación del Mercosur hacia otros países latinoamericanos (por ejemplo mediante la firma de acuerdos de complementación encaminados a crear un área de libre comercio en el Cono Sur latinoamericano que esté en pleno funcionamiento en el curso de la próxima década). ${ }^{4} \mathrm{Y}$ en segundo lugar, el establecimiento de acuerdos de liberalización comercial con la Unión Europea (como el alcanzado en diciembre de 1995) y con el área de libre comercio de Norteamérica (NAFTA) -desarrollando el Acuerdo 4+l firmado con Estados Unidos en junio de 1991 - grupos de países que constituyen los principales socios comerciales del Mercosur.

Con el establecimiento de la unión aduanera el 1 de enero de 1995 (cuyo régimen de excepciones deja fuera del arancel exterior común a los sectores de bienes de capital, informática y telecomunicaciones) ${ }^{5}$ y los avances logrados en la vertebración jurídica del MERCosur, se cierra un primer ciclo en el proceso de inserción en el mercado mundial de las economías que lo componen. La siguiente fase tiene como objetivo genérico la consolidación efectiva del proceso de integración, para lo cual habrá que avanzar mucho en i) la profundización de la apertura externa, ii) el desarrollo

\footnotetext{
${ }^{4}$ Este es el caso de los acuerdos concluidos con Chile y Bolivia durante la cumbre celebrada en la ciudad argentina de San Luis el 2,5 de jumio de 1996. El Mercosur annpliado tiene un PIB conjunto que supera los 900000 miltones de dólares y un mercado potencial de mus de 200 millones de consumidores, lo que lo convierte en uno de los unayores espacios de intercambio comercial y fülanciero del mundo. En el futuro próximo podría establecer acuerdos sìnilares (con velocidades de integración que tengan en cuenta las particularjdades de cada país) con otros paises latinoamericanos. Estas intciativas constituyen la contribución objetiva del Mercosur al logro de una zona de libre comercio hemisférica, como ta planteada en la Cumbre de Miami de diciembre de 1994.

${ }^{5}$ Los bienes sujetos al arancel externo común siponen casi el $85 \%$ del total. El comercio intrarregional de estos bienes está libre de toda barrera arancelaria. Los bienes de capital habrán de converger a un arancel exterior común del $14 \%$ el I de enero del año 2001 en los casos de Argentina y Brasil; Uruguay y Paraguay disponen de cinco años más para adaptar sus aranceles (aumentáudolos progresivamente) al arancel común. Para los sectores de informática y telecomunicaciones los cuatro países establecerán el I de enero del año 2006 un arancel externo común del 16\%. Finalmente, otros bienes (como el azúcar y los productos textiles) están sujetos a regímenes de comercio especiales.
} 
del Mercosur como proyecto estratégico dirigido a la transformación productiva y la inserción competitiva en la economía global (lo que hará necesario dar un salto adelante en la coordinación de las políticas microeconómicas), y iii) la coordinación efectiva y sostenida de las políticas macroeconómicas. Estas tres áreas son claramente interdependientes, por lo que el fracaso en alguna de ellas terminaría imposibilitando el logro de los restantes objetivos.

La coordinación de las políticas microeconómicas y macroeconómicas puede constituir un poderoso estímulo para el crecimiento en la medida en que facili- te profundizar y sostener en el tiempo la apertura económica. La mayor interdependencia económica inducida por los acuerdos de comercio preferencial tiende a potenciar la existencia de externalidades significativas que convierten en subóptimas las decisiones independientes de cada gobierno en un gran número de áreas. La coordinación de las políticas macroeconómicas permitiría evitar (o al menos atenuar) las distorsiones asociadas al diseño discrecional por cada país de sus políticas monetarias y fiscales, con el fin de alcanzar objetivos nacionales independientes de equilibrio interno o externo.

\section{II}

\section{Ganancias de bienestar derivadas de la}

\section{coordinación macroeconómica}

Sin necesidad de recurrir a postulados mercantilistas (que al evaluar los efectos de las políticas nacionales sobre el bienestar no tienen en cuenta las externalidades negativas sobre el resto del mundo, y consideran las relaciones económicas internacionales desde la perspectiva de un juego no cooperativo de suma cero), existe ciertamente una justificación económica para llevar a cabo políticas económicas independientes a nivel de cada nación. Por ejemplo, en una economía plagada de rigideces nominales y asimetrías de información, la no neutralidad del dinero a corto plazo convierte a una política monetaria independiente en un elemento esencial de las políticas de estabilización. Pero una vez establecida la existencia de incentivos para adoptar comportamientos no cooperativos es necesario considerar en qué circunstancias se hace deseable la cooperación macroeconómica, a qué nivel debe realizarse, y cuáles son los obstáculos fundamentales para que el acuerdo cooperativo se alcance y se sostenga a lo largo del tiempo en el marco de un bloque regional que, como el MERCoSur, aspira a convertirse en algo más que un mero acuerdo preferencial.

La justificación económica para formar un verdadero mercado interno a nivel regional residiría, fundamentalmente, en la existencia de bienes públicos o colectivos -incluyendo los objetivos de estabilización, la distribución de la renta, y el entorno de regulaciones- y de diferencias a nivel nacional en las preferencias acerca de dichos bienes (Whitman, 1977, p. 3).
En ese marco, la coordinación de las políticas economicas entrañaría una moditicación signiticativa de las políticas nacionales en respuesta a una interdependencia económica internacional que hace ineficiente la toma independiente de decisiones por parte de los gobiernos, debido a la existencia de externalidades y de bienes públicos internacionales. ${ }^{6}$ El hecho de que el tipo de cambio constituya un vínculo de contacto entre las economías (así como su naturaleza específica de instrumento compartido) podría explicar la importancia alcanzada en la práctica por los acuerdos cambiarios que sustentan la creación o ampliación de las áreas monetarias. La integración de los mercados -la formación de un mercado interior o la realización de una economía integrada- se alcanza cuando (prescindiendo de los costos de transporte) cada tipo de producto o factor de producción se vende a un único precio. Las ganancias de eficiencia asociadas a la economía integrada aumentan a medida que se amplía el tamaño del mercado interior: desde la perspectiva del comercio internacional de bienes y factores productivos

\footnotetext{
- Véase a este respecto Crockett, 1989, p. 345. La paz, la cooperación internacional, la libertad de surcar los nares. los tribunales internacionales de justicia, el sistema de libertad de comercio, un régimen monetario internacional, el sistema financiero internacional, tos patrones de pesos y medidas - incluyendo los teclados y los sistemas operativos compatibles de los ordenadores, los teoremas matemáticos y las monedas de reserva-, constituyen ejemplos habituales de bienes públicos internacionales.
} 
el área económica óptima es el mundo, y cualquier interferencia con el mecanismo de formación de los precios (excluyendo las intervenciones diseñadas para eliminar las imperfecciones del mercado) representará una fuente de ineficiencia en la asignación de los récursos. Sin embargo, este resultado no encuentra su exacto correlato en el tema de las áreas monetarias. ${ }^{7}$

Consideremos, a modo de ilustración, dos países cuyas autoridades monetarias intentan minimizar una función de pérdidas (definida convencionalmente en términos de objetivos de inflación y desempleo) y operan en el marco de una economía pequeña y abierta en la que los precios de los bienes producidos internamente están dados a corto plazo. La consideración explf́cita de las funciones de bienestar es necesaria cuando la política económica persigue más de un objetivo, y cuando - como a menudo sucede - una determinada política tiene efectos positivos sobre algunos objetivos y efectos negativos sobre los restantes. En este conflicto entre objetivos se encuentra el origen del problema de coordinación de las políticas económicas.

Para simplificar la exposición supondremos que existe una perfecta simetría en la estructura de ambos países, que la movilidad del capital es perfecta y que las expectativas con respecto a la variación del tipo de cambio son estáticas y las variables fiscales rígidas a corto plazo, de modo que los efectos de repercusión sobre el otro país de las políticas económicas tienen lugar exclusivamente a través de variaciones en el nivel del tipo de cambio. El apéndice que figura al final dei trabajo describe la estructura de la economía -ecuaciones [A.1] a [A.6] - y desarrolla los rasgos esenciales del modelo. ${ }^{8}$ Supongamos a continuación que los dos países se ven afectados por una perturbación de precios similar que hace aumentar en la misma magnitud sus

\footnotetext{
${ }^{2}$ La teoría de las áreas monetarias óptimas sugiere que, a medida que aunxenta el tamnāo del área monetaria, aumentan los ganancias derivadas de la estabilidad cambiaria, aunque lo hacen a una tasa decreciente. Al mismo tiempo, la ampliación del área monetaria aumenta la probabilidad de que se produzca un muyor número de perturtaciones asimétricas y, en consecuencia, la probabilidaal de que se generen pérdidas de bienestar a un rituno creciente. Por lo tanto, el área monetaria óptima no es el mundo: es posible, pues. proveer una justificación económica para el mantenimiento de un cierto grado de la mal llamada —abusando de una jerga con resonancias manifiestamente mercantilistas- soberanía monetaria.

${ }^{8}$ Los trabajos de Hamada (1976 y 1979) constituyen la referencia básica para el análisis de los problemas de ccordinación que se plantean en el marco de diferentes regímenes cambiarios. Para una excelente introducción a los problemas generales de la coordinación macroeconómica con aplicaciones al caso del Mercosur, véanse los trabajos de Heyman y Navajas (1992) y Ferrer y Lavagia (1992).
}

tasas de inflación. En el concexto de nuestro modelo la política monetaria sólo puede influir en la tasa de inflación a través del tipo de cambio, por lo que este instrumento se convierte en el objetivo intermedio de la política antiinflacionista en ambos países. La solución no cooperativa de Cournot-Nash se determina suponiendo que las autoridades monetarias de cada país toman las decisiones de política monetaria, orientadas a minimizar su función de pérdidas -que describe la ecuación [A.12]--, considerando como dada la política monetaria del otro país (es decir, tratando el acervo monetario del otro país como un parámetro).

El juego no cooperativo se resuelve necesariamente con una contracción monetaria excesiva en ambos países. Los resultados principales vienen descritos por las expresiones [A. 15] a [A.19]. Ambas autoridades monetarias compiten para aumentar su reputación antiinflacionista ( $y$ la credibilidad de su política monetaria), instrumentando políticas monetarias contractivas. Pero la contracción monetaria no consigue en el equilibrio de corto plazo modificar la tasa de inflación en ninguno de los dos paŕses, dado que los bancos centrales de ambos intentan modificar la misma variable nominal en direcciones opuestas, contrarrestando cada uno con su intervención los logros iniciales del rival. La no coordinación de las políticas monetarias tiene consecuencias reales negativas para ambos países: la política de apreciaciones competitivas se acabará traduciendo en un aumento de los tipos de interés reales y en una caída de la producción por debajo del nivel deseado en cada país. ${ }^{9}$

Estos costos reales pueden evitarse mediante un acuerdo cooperativo de ambas autoridades monetarias. El resultado cooperativo es equivalente al que se alcanza cuando un comité monetario común resuelve el problema de optimización global descrito por la ecuación [A.20]. Dado el conflicto entre los objetivos de ambos países, el comité monetario se abstendrá de utilizar el tipo de cambio como objetivo intermedio de

\footnotetext{
${ }^{9}$ Para simplificar la exposición, en el apéndice nos abstenemos de analizar la solución correspondiente al equilibrio no cooperativo de Stackelterg-Nash. La solución de Stackelberg-Nash implica que la tasa de variación del tipo de caunbio es distinta de cero, por lo que la condición de arbitraje de intereses $\left(i=i^{*}\right)$ iene que ser revisada y el modelo resuetto bajo las nuevas condiciones. En este caso el líder utiliza la información disponible sobre el otro país (materializada en su función de reacción) para sobreexpandir $m$, dado que sabe (a la vista de la información suministrada por la función de reacción del seguidor) que las autoridades del otro país intentarán apreciar su moneda a través de una contracción monetaria. De este modo, el comportamiento del gobjerno que ejerce el liderazgo podría permitir limitar la contracción global que se sufre en el equjlibrio de Cournot-Nasin.
} 
su política antiinflacionista. De hecho, el equilibrio resultante implica que los gobiernos se ponen de acuerdo para aceptar la tasa de inflación inicial y concentran sus esfuerzos en disminuir la varianza de la producción alrededor del equilibrio de pleno empleo (ecuaciones [A.21] y [A.22]), dado que en este caso $m=m^{*}=q=q^{*}=p_{0}$. En el contexto de este juego estático (de un solo período), el bienestar que se alcanza bajo el equilibrio cooperativo es claramente superior al que se obtiene en el caso de Cournot-Nash: la tasa de inflación es similar en ambos, pero la desviación de la producción se minimiza (es igual a cero) en la solución cooperativa. Por el contrario, el equilibrio no cooperativo de Cournot-Nash implica que $m=m^{*}$ $<q=q^{*}=p_{0}$, abriendo la posibilidad a una reducción progresiva (si se aceptan los costos de ajuste) de la tasa de inflación del área monetaria. ${ }^{10}$

El acuerdo cooperativo inicial no es suficiente por sí mismo para modificar la estructura de la economía (flexibilizando los salarios y reduciendo el grado de indización salarial) en la medida necesaria para facilitar el proceso de desinflación. Si las autoridades mo- netarias persisten en su voluntad de reducir la tasa de inflación de ambos países a lo largo del tiempo, sería necesario complementar el acuerdo cooperativo -en el marco de un juego esencialmente dinámico- con criterios adicionales de convergencia nominal, dado que en nuestro modelo la cooperación se traduce en un acuerdo expreso para sostener la tasa de inflación inicial. Además, hay que tener en cuenta que la convergencia nominal es una condición necesaria, pero no suficiente para alcanzar la convergencia real. Por to tanto la coordinación macroeconómica debe verse reforzada con la puesta en marcha de reformas estructurales dirigidas a tlexibilizar los mercados (especialmente en el sector servicios y en el mercado de trabajo). En definitiva, un acuerdo monetario satisfactorio no puede ser definido solamente en términos de reglas que gobiernan el tipo de cambio: los instrumentos utilizados para mantener el compromiso cambiario tienen repercusiones tanto en el bienestar de cada país como en la estabilidad del sistema. Por consiguiente, la mera fijación del tipo de cambio no resuelve el problema general de los efectos externos."

\section{III}

\section{Fórmulas de coordinación macroeconómica}

Cuando las autoridades deciden cooperar modificando sus políticas económicas de manera mutuamente beneficiosa teniendo en cuenta las interdependencias existentes, deben acordar, en primer lugar, cuál será la extensión de la cooperación. Una coordinación absoluta abarcaría todas las instancias de la política macroeconómica (incluyendo los objetivos finales y los instrumentos monetarios, fiscales, y otros de que dis-

\footnotetext{
:Esta reducción de la inflación depende de factores que no se uspecifican en et modelo simplificado, y que básicamente relacioutan la reducción de ta producción con la disminución de las tasas de inflación. Para analizar brevemente esta cuestion consideremos una ampltación de la estructura del modelo, incorporando una ecuacion de precios $p=w$, obtenida a partir del supuesto de que las tmpresas determinan los precios estableciendo un margen sobre los costos medios variables, y una ecuación de salarios, $w=\delta q+\phi y$ (donde $w$ representa la tasa de crecimiento de los salarios, el parametro $\delta$ refleja el grado de indización salarial y el parámetro $\phi$ refleja el grado de flexibilidad salarial de la economia). Sustituyenclo la ecuación de salarios en la ecuación de precios obtenemos la siguiente expresión, $p=\delta q+\phi y$. De modo que, en un contexto clinámico, la reducción transitoria de la producción posibilita la caída cle la inflación a largo plazo.
}

pone cada gobierno). La coordinación debe basarse necesariamente en el establecimiento de compromisos para las acciones futuras de política económica, lo que nos lleva a formular dos preguntas inevitables: i) itie-

\footnotetext{
" En entornos conceptuales similares Krugman (1990) y McKibbin y Sachs (1991) muestran que la comparación entre ef equilibrio cooperativo y el no cooperativo, en términos de las ganancias de bienestar resultantes a largo plazo, depende esencialmente de los objetivos de los gobiemos y de los fijadores de salarios, así como de las interacciones de ambos. En la misma línea Oudiz y Sachs (1986) sef̂alan que, en general, la coordinacion internacional resulta menos deseable en los modelos dinámicos que en los modelos estáticos de un período. Sin embargo, estos autores producen un ejemplo que muestra que la cooperación es preferible también en un contexto dinámico porque permite eliminar la inconsistencia temporal que earacteriza a las políticas monetarias en un régimen no cooperativo. Pero ese resultado se alcanza a partir del supuesto de que las autoridades poseen una capacidad de compromiso absoluta en cuanto a las acciones de política futuras. La eficiencia relativa de la solución no cooperativa frente a la imposición de criterios de convergencia rominal destinados al control de la inflacion (en el marco de la solución cooperativa propiciada por el acuerdo cambiario), es una cuestión ambigua que depende del entorno conceptual en el que se desarrolla el análisis.
} 
nen los gobiernos capacidad de compromiso suficiente para asegurar un comportamiento consistente en el tiempo?; y ii) ¿tienen los gobiernos capacidad de control suficiente para modificar los instrumentos cuando ello resulta necesario para preservar el acuerdo cooperativo? La respuesta es obviamente negativa en ambos casos (incluso en una unión económica y monetaria, en vista de las dificultades para coordinar las políticas fiscales). En consecuencia, la coordinación macroeconómica tiene que ser necesariamente parcial, limitándose por lo general a aspectos concernientes a las posiciones relativas de los países. Esto contribuye a explicar la importancia que habitualmente se concede a la balanza de pagos y al tipo de cambio en la teoría y la práctica de esa coordinación.

El hecho de que la coordinación macroeconómica tenga un carácter parcial implica que algunos objetivos se fijan independientemente (de manera discrecional $\mathrm{u}$ obedeciendo reglas internas) y que algunos instrumentos están al margen de la negociación. Sin embargo, los grados de libertad de que disfrutan las autoridades en la práctica pueden ser mucho menores de lo que sugiere la relatividad de los acuerdos. Las restricciones intertemporales crean vínculos indisolubles entre las políticas monetarias y fiscales, de modo que frecuentemente es necesario establecer un grado minimo de coordinación fiscal (por ejemplo, a través de programas de convergencia que limiten los déficit presupuestarios) para lograr que la coordinación monetaria sea efectiva. Por otra parte, el problema crucial de la consistencia temporal de las políticas sugiere la necesidad de complementar la cooperación con reformas institucionales de alcance con el fin de garantizar la sostenibilidad de los acuerdos. ${ }^{12}$

Los acuerdos de cooperación tienen lugar inevitablemente en un entorno de incertidumbre. En estas circunstancias las ganancias de bienestar que se derivan $e x$ ante del acuerdo pueden tornarse en pérdidas de bienestar cuando el programa se ha ejecutado, debilitando los incentivos para que los gobiernos cooperen. La incertidumbre tiene su origen en la existencia de errores de información y en las perturbaciones exógenas que afectan al sistema, pero también existe incertidumbre relativa a la calidad de los modelos utilizados para el análisis, a las verdaderas preferencias

\footnotetext{
${ }^{12}$ Por ejemplo, un país podría elevar al rango de ley fundainental la obligación de mantener un presupuesto equilibrado, prohibiendo en todo caso la monetización de los déficit y concediendo al banco central un estatuto de independencia que inmunice a las políticas monetarias frente a los desequilibrios presupuestarios.
}

(objetivos y prioridades) de las autoridades, y a la sostenibilidad de los acuerdos. La coordinación macroeconómica en condiciones de incertidumbre puede ser especialmente beneficiosa (puede reducir los errores de la política) si la cooperación permite internalizar sus efectos y distribuir los riesgos entre los países participantes en lugar de promover el desplazamiento de éstos hacia los competidores. ${ }^{13}$

La coordinación de las políticas implica como mínimo un intercambio de información entre las partes; el siguiente escalón consiste en negociar el tipo de regla que las autoridades se comprometen a seguir; finalmente las autoridades pueden adoptar un acuerdo que involucre todos los aspectos de la política común. Cualquiera que sea el nivel de la cooperación, en un contexto dinámico resulta esencial asentar la reputación de las autoridades para dotar de credibilidad a los acuerdos. La estrategia adoptada para cimentar tal reputación determina los efectos de esta política sobre el nivel de bienestar: el modelo de la sección anterior proporciona un ejemplo de cómo las actuaciones no cooperativas de cada gobierno para mejorar su reputación a través del instrumento común (el tipo de cambio) pueden terminar produciendo una pérdida neta de bienestar. Este es probablemente uno de los resultados mejor establecidos en lo que se ha escrito sobre coordinación macroeconómica.

La coordinación macroeconómica se concreta frecuentemente en la elección de, al menos, una variable objetivo intermedio cuya regulación permita el control de los objetivos finales (representados por metas de inflación y crecimiento). En este contexto resulta apropiado definir un ancla nominal como una variable nominal que el gobierno decide convertir en el objetivo intermedio de la política. Por supuesto, la primera condición para que la política sea eficaz es que se centre en el logro de objetivos que estén a su alcance. $\mathrm{Si}$ el gobierno (actuando independientemente o en el marco de un acuerdo cooperativo) pretende fijar al margen del mercado una variable real (por ejemplo la tasa de desempleo, el tipo de interés o el tipo de cambio real), no sólo no conseguirá sus objetivos sino que inevitablemente conducirá a la economía a un círculo vicioso que terminará en una espiral inflacionaria.

\footnotetext{
${ }^{13}$ Las estimaciones empíricas de las ganancias de bienestar de la coordinación macroeconómica muestran grandes oscilaciones como consecuencia de las diferencias en los modelos de referencia, en las funciones objetivos utilizadas y en los supuestos acerca de la credibilidad de las políticas. Véase un examen reciente de las publicaciones sobre este tema en Currie, 1993.
} 
El papel de ancla nominal puede ser bien desempeñado por variables monetarias o por el tipo de cambio. ${ }^{14}$ La utilización de un ancla monetaria implica que la política monetaria fija el precio de un bien o grupo de bienes en términos de la moneda del país (el ejemplo más notable es el patrón oro) o, alternativamente, que el objetivo intermedio de la política monetaria es la tasa de variación de una magnitud nominal (el nivel de precios, un agregado monetario determinado, o la renta nominal). La utilización del tipo de cambio como ancla nominal también presenta numerosas variantes: la autoridad monetaria puede fijar el nivel o la tasa de variación del precio de la moneda de un país en términos de una moneda extranjera de referencia, o puede establecer una banda de fluctuación alrededor de una paridad central fija, o puede intervenir con mayor o menor frecuencia en los mercados de divisas para controlar la evolución del tipo de cambio. ${ }^{15}$

\section{IV}

\section{La selección del ancla nominal}

La selección del ancla nominal que servirá de fundamento a la coordinación macroeconómica es un proceso muy complejo en el que influyen factores como la transparencia de la variable nominal elegida, la capacidad de control de la misma por parte de la autoridad monetaria, o la estabilidad de la relación entre el ingreso real y la variable nominal que opera como objetivo intermedio. La naturaleza de las perturbaciones que afectan al sistema también resulta crucial para determinar la optimalidad relativa de un acuerdo cooperativo. El ajuste de una economía afectada por una perturbación real de carácter asimétrico requiere un cambio de los precios relativos de los bienes y factores del país con respecto al resto del mundo, cualquiera sea el régimen cambiario vigente. Si la estructura de la economía genera una no neutralidad del dinero a corto plazo, la flexibilidad del tipo de cambio nominal permite que los ajustes necesarios se produzcan más rápidamente y con menores costos de ajuste. $\mathrm{Si}$, por el contrario, predominan las perturbaciones de carácter nominal (por ejemplo, perturbaciones fïnancieras intensas que desplacen erráticamente la deman-

\footnotetext{
14 Véase un análisis más detallado del papel del tipo de cambio como ancla nominal en los trabajos de Giovannini (1988), Quirk (1996) y Rodríguez Prada (1996).

is Por otra parte, ta balanza de pagos puede sustituir at tipo de cambio como objetivo intermedio de la política económica y el conflicto entre objetivos puede resurgir con virulencia en un régimen de tipos de cambio fijos. Por ejemplo, si cada país tuviese un objetivo independiente para el balance en cuenta corriente el sistema de tipos de cambio fijos se vería abocado -en ausencia de una corrección fiscal o monetaria suficiente- a sufrir una presión deflacionista zontinua, sin que los objetivos indjviduales de alcanzar un superívit corriente llegaran a materializarse. En un escenario alternativo, en el que los países se mostrasen indiferentes acerca de los efectos
}

da de dinero), la flexibilidad del tipo de cambio nominal inducirá ajustes de los precios relativos excesivos e innecesarios y con costos de ajuste reales potencialmente importantes. ${ }^{16}$

La flexibilidad del tipo de cambio será deseable en el caso de que predominen las perturbaciones reales asimétricas en cada país, tanto transitorias como permanentes. Los cambios asincrónicos del ciclo económico proporcionan un buen ejemplo de perturbaciones reales asimétricas de carácter transitorio. Supongamos, por ejemplo, que Brasil experimenta una fuerte recesión. La reducción del nivel de producción dará lugar a un exceso de oferta de dinero (como consecuencia de la caída de la demanda de saldos reales) que reducirá los tipos de interés, creando un diferencial de intereses desfavorable para Brasil que forzará un incipiente flujo de capitales hacia el exterior. Sin ataduras para el tipo de cambio, el real se depreciaría en la cuan. tía necesaria para generar expectativas de apreciación suficientes para restaurar la condición de arbitraje en los mercados financieros internacionales. La depreciación del real ejercería a lo largo del tiempo una función de estabilizador automático de la economía, al estimular la producción de bienes transables y elevar la demanda agregada mediante el crecimiento de las exportaciones netas.

Incluso en el caso de que la perturbación real asimétrica tuviese carácter permanente el ajuste del tipo

de sus políticas sobre los balances en cuenta corriente, éstos tende-
rían a poner en marcha políticas fiscales o monetarias demasiado
expansivats que redundartan en déficit corrientes excesivos.
l' Estos resultados se obtienen a patir de una sencilla extrapolación
(al caso de una economía abierta) del clásico trabajo de Poole, 1970. 
de cambio podría aportar grandes beneticios. Supongamos, por ejemplo, que la economía brasileña sufre una crisis estructural que se manifiesta en la decadencia de la espina dorsal industrial en la que el país se ha especializado. De nuevo, con una regla monetaria constante, la progresiva reducción de la competitividad terminaría produciendo una reducción del tipo de interés real que se traduciría en una disminución del costo de oportunidad de las inversiones y del endeudamiento a largo plazo. El diferencial de intereses resultante constituiría un primer incentivo para un desplazamiento de la inversión internacional hacia Brasil que mitigase los esfuerzos derivados de la necesaria reconversión industrial.

Sin embargo, en un escenario tan adverso de crisis industrial posiblemente haría falta algo más que un abaratamiento del crédito para estimular la inversión. Habría que generar además una baja en el precio relativo de los activos reales brasileños (fundamentalmente de los bienes de capital, inmuebles y tierras) que fuese suficiente para atraer la inversión extranjera directa necesaria. Cabe preguntarse si en este contexto puede aportar algo la flexibilidad cambiaria. Con tipos de cambio fijos el ajuste de los precios relativos habría de realizarse forzosamente mediante una caída de los precios nominales de estos activos reales. Una reducción significativa del valor en moneda nacional de los activos reales brasileños haría disminuir el valor de la garantía que respalda el crédito proporcionado por el sistema financiero, poniendo en peligro la solvencia de las entidades financieras y motivando, si la crisis es suficientemente intensa, la intervención del banco central como prestamista de última instancia.

La depreciación del real facilitaría en gran medida el ajuste en las anteriores circunstancias, dado que la reducción requerida en términos reales en los precios de los activos se lograría en buena parte mediante el ajuste del tipo de cambio. Tras esa depreciación el valor en dólares de los activos brasileños sufriría una cafda relativamente intensa, pero su valor en reales permanecería prácticamente inalterado. Por el contrario, con tipos de cambio fijos, Brasil necesitaría recutrir a una drástica deflación para lograr la misma merma en el valor en dólares de sus activos. El análisis anterior presupone que a corto plazo no se satisface la hipótesis de la paridad del poder de compra, de modo que las variaciones del tipo de cambio nominal se reflejan, al menos durante varios meses, en el tipo de cambio real. En un escenario de recesión económica ese suele ser el caso. ${ }^{17}$ ?

Por otra parte hay que tener en cuenta que, como la experiencia de Argentina demuestra, incluso en países que parten de una situación de hiperinflación y que se ven afectados por una dolarización elevada, la fijación del tipo de cambio no conlleva una fijación inmediata del índice general de precios. ${ }^{18}$ Incluso si el país logra reconducir el proceso inflacionario a tasas de estabilidad perfectamente equiparables a las existentes en países industrializados en un período de dos o tres años, la apreciación real de la moneda puede generar en poco tiempo importantes déficit en las cuentas externas. ${ }^{19}$ De hecho, la utilización de un ancla cambiaria en un entorno de libre movilidad del capital puede generar episodios inflacionatios relativamente intensos. El origen de este fenómeno se encuentra en las dificultades de practicar una política efectiva de esterilización de las reservas con el fin de contrarrestar el efecto expansivo sobre las magnitudes monetarias de los flujos de capitales (atraídos por el entorno más estable propiciado por una mayor credibilidad de la política monetaria). La esterilización de las reservas implica incrementos de los tipos de interés que dan un renovado impulso a las entradas de capitales, dificultando el control monetario. ${ }^{20}$

\footnotetext{
${ }^{17}$ Las depreciaciones nominales y reales experimentadas, por ejennplo, por la libra esterlina, la lira y la peseta tras la crisis del Sistema Monetario Europeo (SME) en septiembre de 1992, son un ejemplo contundente al respecto, sobre todo teniendo en cuenta que la convergencia a la baja de las tasas de inflación (especialnente en el sector de bienes no transables) se aceleró precisamente tras las devaluaciones.

Ix En Argentina la inflación acumulada durante los dos afos que siguieron a la implantación del Plan de Convertibilidad (desde abril de 1991 a mayo de 1993), medida por el índice de precios al consumidor, aumentó en un $46 \%$, mientras que en Brasil, en los dos años que siguieron a la puesta en marcha del Plan Real (desde julio de 1994 a junio de (996), el incremento acumulado de los precios al consumidor se situó en torno al 55\%. Véase un sucinto análisis de los problemas asociados a la estabilización de una hiperinflación, con abundantes referencias a América Latina, en Heyman (1992). ${ }^{19}$ Por ejemplo Brasil tuvo en 1996 un déficit comercial de unos 5500 millones de dólares (cifra sin precedentes), mientras que su déficit en cuenta corriente superó los 24000 millones de dólares (el $3.3 \%$ del PiB). Las proyecciones (realizadas a principios del aîio) estiman que en 1997 se alcanzatá un déficit comercial cercano a los 8000 millones de dólares.

20 Como casos ilustrativos, Estonia padeció un atumento transiforio de la tasa de inflación poco después de una drástica reforma monetaria (tras abandonar el área del rublo y transformar su banco central en una cuasi caja de conversión), y Egipto suftió fuertes presiothes inflacionistas luego de la guerra del Golfo.
} 


\section{La coordinación macroeconómica}

\section{posible en el Mercosur}

Las economías que componen el Mercosur son demasiado distintas entre sí para que sea factible una coordinación estricta de las políticas macroeconómicas (el problema se acrecienta si se contempla un Mercosur ampliado a Chile y Bolivia). La provisión optima de bienes públicos y colectivos -incluyendo los objetivos de estabilización, la distribución del ingreso, y el entorno legal e institucional- determina la dimensión óptima del bloque regional (Cooper, 1976). Cualquier proyecto de coordinación de las políticas económicas en el Mercosur tiene que tener en cuenta las realidades estructurales e institucionales del bloque regional. La existencia de asimetrías difícilmente reversibles en el corto plazo (como el tamaño del mercado, el nivel de industrialización, el grado de apertura, el ingreso per sápita, el grado de dolarización, etc.), genera un rechazo instintivo a la excesiva homogeneidad de las soluciones. ${ }^{21}$

Mundell (1961), tomando prestada la distinción icardiana entre comercio interno y comercio exterior íperfecta movilidad de los factores dentro de cada país y ausencia de movilidad entre países), mostró que en un área monetaria 6ptima la existencia de un alto grado de movilidad de los factores productivos (o la existencia de flexibilidad salarial suficiente), sustituye a las variaciones del tipo de cambio como instrumento de ajuste. La discontinuidad en el grado de movilidad del trabajo (no explicada ni por Ricardo ni por Mundell) podría atribuirse en parte (además de a los costos de transporte) a la heterogeneidad lingǘstica y cultural entre sociedades diversas. En el MERCosUR, todavía en una fase de integración incipiente, la necesaria movilidad intrarregional de los factores productivos es aún muy escasa. Además, el grado de apertura, el tamaño medio y el grado de diversificación de las economías del Mercosur no permiten descartar la utilización ocasio-

\footnotetext{
${ }^{21}$ En Argentina más de la mitad de los depósitos del sistema financiero se encuentran denominados en dólares. La dolarización es cificilmente reversible y parece aumentar inciuso en un entorno de aparente convergencia nominal (el caso de Bolivia, con una colarización del sistena financiero cercana al $90 \%$ tras más de una cécada de estabilidad macroeconómica, es un buen ejemplo).
}

nal del tipo de cambio para restaurar la competitividad perdida. 22

Si las perturbaciones internas que sufre el bloque regional son relativamente pequeñas en comparación con las externas, la política óptima consistiría en: i) favorecer una separación de los riesgos, intentando aislar a la región del mundo exterior mediante la aplicación de barreras comerciales y controles cambiarios, $y$ ii) reforzar el mercado interno, adoptando una moneda común a nivel del bloque regional. Si las perturbaciones internas y externas son de magnitud similar pero están imperfectamente correlacionadas, la política óptima sería la de difundir los riesgos mediante la integración económica y monetaria de ambas partes. Finalmente, si las perturbaciones que afectan a las distintas regiones del área monetaria tuviesen efectos similares, la política monetaria apropiada sería la misma en cada región y no se ganaría nada con una mayor flexibilidad de los tipos de cambio. Sin embargo, muchas de las perturbaciones son específicas (idiosincráticas) de cada país, y las de origen común tendrán en general efectos asimétricos al incidir sobre estructuras económicas diferenciadas. Si la asimetría de las perturbaciones prevalece, la fijación de los tipos de cambio entrañaría un sacrificio probablemente muy grande de una política monetaria potencialmente beneficiosa. ${ }^{23}$ En conjunto, las diferencias en las estruc-

\footnotetext{
${ }^{22}$ A medida que aumenta el grado de apertura, la flexibilidad del tipo de caunbio resulta menos efectiva para ajustar el balance en cuenta corriente ante perturbaciones reales de la demanda, puesto que las variaciones del tipo de cambio nominal se trasladarán más rápidamente a los precios de los bienes, limitando el ajuste del tipo de cambio real (McKinnon, 1963). Por otra parte un elevado grado de diversificación hace menos necesario el ajuste del tipo de canbio para absorber perturbaciones específicas de un sector productivo (Kenen, 1969).

${ }^{23}$ Por ejemplo, Feldstein ( 1992) ha criticado el objetivo de la unión monetaria europea aduciendo que la actual Unión Europen no es un área monetaria optima porque la movilidad del trabajo es escasa y la existencia de perturbaciones asimetricas continúa haciendo necesario el ajuste del tipo de cambio. Véase un punto de vista distinto, radicalınente favorable a la unión monetaria, en Buiter (1995). Para este autor el grado de convergencia real es irretevante para la unión monetaria en la medida en que las rigideces de los mercados (por ejemplo las rigideces salariales) no pueden verse aliviadas por ta flexibilidad cambiaria ni por la independencia monetaria.
} 
turas económicas subyacentes de los países que conforman el Mercosur aumentan la probabilidad de que prevalezcan las perturbaciones idiosincráticas y de que las comunes tengan efectos asimétricos.

En el caso específico del Mercosur las perturbaciones están asimétricamente distribuidas y las velocidades de ajuste varían de un país a otro, haciendo necesaria la modificación ocasional del tipo de cambio para disminuir el costo del proceso de ajuste. Bayoumi y Eichengreen (1994) han mostrado que las tensiones cambiarias entre Argentina y Brasil reflejan la existencia de perturbaciones de demanda diferenciadas, y que en la economía brasileña es más baja la velocidad de ajuste de la producción a las perturbaciones, por lo que una política monetaria estrictamente orientada a la estabilización del tipo de cambio bilateral tendería a agravar los costos del ajuste. Refuerza este resultado el hecho de que las perturbaciones de oferta están débilmente correlacionadas entre los países del Mercosur (especialmente en el caso de Uruguay y Paraguay). ${ }^{24}$ Por otra parte, las perturbaciones de oferta en el caso de Argentina y Chile están inversamente correlacionadas con las de Estados Unidos. Este resultado hace todavía más difícil justificar el mantenimiento a largo plazo de un tipo fijo entre el peso y el dólar, y abona la implantación de un régimen cambiario relativamente más flexible.

El establecimiento de una banda cambiaria constituye una alternativa ( $y$ proporciona una estrategia de salida en el marco de un acuerdo cooperativo) a la utilización del tipo de cambio como ancla nominal. Un mecanismo de bandas de fluctuación supone un compromiso no lineal entre un sistema de tipos de cambio tijos y un sistema de tipos de cambio flexibles. Básicamente consiste en la definición de un régimen cambiario que permite explotar los grados de libertad proporcionados por tipos de cambio que fluctúan libremente, sin renunciar por ello a la coordinación de las políticas monetarias que aporta un régimen de tipos de

\footnotetext{
${ }^{24}$ El mismo estudio muestra que en el caso del NaFTa tampoco se dan las condiciones para una unificación del tipo de cambio, ya que existe una correlación inversa significativa de las perturbaciones de oferta de Estados Unidos con respecto a los de Canadá y México. Además, las perturaciones de demanda que afectan a Estados Unidos y México muestron una fuerte correlación negativa (mientras que la correlación entre Estados Unidos y Canadá resulta insignificantemente positiva).
}

cambio fijos. ${ }^{25}$ En estos modelos, la anticipación de intervenciones defensivas en el momento de la colisión del tipo de cambio con los límites de la banda genera importantes no linealidades que se reflejan en la curvatura de la trayectoria típica - -una curva en forma de $S$ - que sigue el tipo de cambio en el interior de la banda. La connotación esencial es el carácter estabilizador del sistema (de modo que los cambios en las variables macroeconómicas fundamentales tienen un efecto menor sobre los tipos de cambio en un régimen de bandas de fluctuación que en uno de tipos flexibles).

Las dificultades para calcular el tipo de equilibrio constituyen en sí mismas un argumento adicional a favor de un sistema de bandas de fluctuación. La paridad central puede revisarse periódicamente con el fin de evitar alejamientos prolongados con respecto al tipo de cambio de equilibrio de largo plazo. ${ }^{26} \mathrm{Si}$ los agentes confían plenamente en la capacidad del gobierno para mantener el tipo de cambio dentro de la banda. este tipo de mecanismo cambiario exhibe claras propiedades estabilizadoras. Sin embargo, en presencia de incertidumbre, cuando la probabilidad de que se produzca un realineamiento supera un determinado umbral las propiedades estabilizadoras de la banda se transforman abruptamente y el mecanismo de bandas de fluctuación se hace intrínsecamente inestable, propiciando un ataque especulativo. En general, en un contexto de incertidumbre, el aumento de la frecuencia de los realineamientos y el aumento de la amplitud de la banda elevan los riesgos que asumen los especuladores y, en consecuencia, contribuyen a restaurar la credibilidad del mecanismo de tipos de cambio.

\begin{abstract}
${ }^{25}$ El modelo seminal de Krugman (1988) mostró de manera muy elegante la capacidad de este régimen cambiario para estabilizar el tipo de caunbio nominal dentro de la banda, racionalizando de este modo la utilización de mecanismos cambiarios como el que usa en el sme. Este mnodelo está basado en un modelo monetario simple de determinación del tipo de cambio que incorpora una serie de supuestos extremadamente restrictivos: completa flexibilidad de precios; perfecta credibilidad de la banda; defensa de la banda mediante intervenciones marginales infinitesimales, y una especificación de los fundamentos macroeconónicos demasiado simple, pero técnicamente útil para encontrar una solución explícita del modelo. ${ }^{26}$ Williamson y Miller (1987) acuĩaron el térınino de "zona objetivo" para designar un mecanismo de bandas de fluctuación definido en torno al tipo de catmbio real, que a su juicio debería servir de base a la coordinación macroeconomica entre los países del Grupo de los Siete.
\end{abstract}




\section{Un modelo centro-periferia de}

\section{coordinación monetaria}

Como se desprende del análisis realizado en la sección anterior, un somero examen de las características del Mercosur basta para rechazar a priori la conveniencia de fundamentar la coordinación macroeconómica entre los países del entorno del Mercosur en un estricto régimen de tipos de cambio fijos. Sin embargo es preciso reconocer que las realidades institucionales refuerzan el papel del dólar en todas las economías del Mercosur, y especialmente en Argentina, donde la Ley $\mathrm{N}^{\circ} 23928$ de Convertibilidad de abril de 1991 transformó el banco central en una cuasi caja de conversión (currency board) (que respalda la base monetaria con divisas convertibles y acepta una convertibilidad sin restricciones de la moneda del país por la moneda externa de referencia), y fijó un tipo de cambio de un peso por dólar. ${ }^{27}$ Por otra parte, los temores a las repercusiones de un cambio de régimen sobre los mercados financieros han creado la percepción en amplios sectores de ta sociedad argentina de que los costos de salir del sistema de convertibilidad serían muy elevados. Dado que Brasil también ha adoptado desde hace algún tiempo una estrategia de desinflación basada en el uso del tipo de cambio como ancla nominal, ${ }^{28}$ resulta de interés investigar las propiedades básicas (y las limitaciones) de un mecanismo de coordinación macroeconómica que se apoya en un modelo centroperiferia de coordinación monetaria basado en el dó

\footnotetext{
7 Véanse análisis económicos recientes del funcionamiento de las cuasi cajas de conversión que toman en consideración la experiencia argentina, en los trabajos de Connolly (1995), Judy (1995), Williamson (1995), Zarazaga (1995) y Rodríguez Prada (1994, 1995 1996).

3x. Tras la puesta en marcha del Plan Real, Brasil también ha venido utilizando una banda cambiaria implícitamente basada en el dólar. La segunda fase del programa brasileño de reforma monetaria (pretedida por una política de ajuste fiscal) consistio en la introducción en marzo de 1994 de una unidad real de valor (URv) pegada al dólar que serviría como unidad de cuenta y de referencia para todos los contratos; su objetivo era reducir radicalmente la inflación inercial ile una economía que, gracias a la masiva indización, se había acostumbrado a convivir con la hiperinflación sin recustir a la dolarización. El I de julio de 1995 la URV adquirió las propiedades de unidad de cuenta, medio de pago y depósito de valor, transformán. close en el real y completando la reforma monetaria. Véase en Sachs y Zini (1996) una evaluación reciente del Plan Real.
}

lar. El modelo también ayuda a racionalizar la percepción habitual de que los países de la periferia son seguidores naturales.

El argumento convencional para justificar la utilización de un ancla cambiaria es que las instituciones monetarias de to que llamaremos periferia carecen de la suficiente reputación antiinflacionista, lo que obliga al gobierno a "tomar prestada" un ancla monetaria externa mediante la fijación del tipo de cambio. En efecto, la fijación del tipo de cambio en un entorno caracterizado por una elevada movilidad del capital inhabilita a la autoridad monetaria de la periferia para perseguir una política monetaria independiente, de modo que "hereda" automáticamente la política monetaria del centro. El modelo se centra exclusivamente en el sector monetario. Las variables correspondientes al centro se representan con un asterisco y las ecuaciones de ambos países tienen una estructura simétrica (se omiten inicialmente, para simplificar la notación, las ecuaciones correspondientes al centro). Las siguientes ecuaciones describen el mercado monetario de la periferia: ${ }^{29}$

$$
\begin{aligned}
& M=-n i+\mu \\
& M=C I+R \\
& C I=D-f R, \quad 0 \leq f \leq 1
\end{aligned}
$$

\footnotetext{
${ }^{29}$ La demanda de dinero -ecuación [ $[1]$ - depende del tipo de interés $i$, y (con elasticidad unitaria) de una perturbación exogena $\mu$ sobre la velocidad. Para simplificar, supondremos que la elasticidad de la demanda de dinero cont respecto al tipo de interés es la misma en ambos países $\left(n=n^{*}\right)$. La ecuación [2] muestra la coinposición del acervo monetario $M$, integrado por el crédito interno $C I$ y tas reservas de djvisas $R$. El mantenimiento det control monetario puede exigir la esterilización de las reservas: el banco central puede esteritizar el efecto (sobre el acervo monetario) de su intervención en los mercados de divisas, interviniendo simultáneamente en el mercado monetario interno. Por ejemplo, al tiempo que compran moneda extranjera en el mercado de divisas, las autoridades monetarias pueden realizar una operacion de mercado abjerto consistente en la venta de bonos con el fin de reducir el crédito interno. La ecuación [3] refleja la regla de esterilización seguida por tas autoridades monetarias: $D$ es el componente autónomo (exógeno) del crédito intemo, y el parómetro $f$ es el coeficiente de esterilización.
} 
En condiciones de perfecta movilidad del capital, la condición de arbitraje de intereses (equilibrio de cartera) es

$$
i=i^{*}+\lambda
$$

donde $\lambda$ representa la existencia de una perturbación exógena sobre las preferencias de cartera de los mantenedores de riqueza. Para cerrar el modelo es necesario especificar la regla de intervención en el mercado de divisas:

$$
\theta=R /\left(R \cdot R^{*}\right) \quad 0 \leq \theta \leq 1
$$

La solución del modelo proporciona las siguientes ecuaciones en forma reducida:

$$
\begin{aligned}
& M=(I-\varepsilon) D+\varepsilon \cdot D^{*}+\varepsilon \cdot \omega \\
& M^{*}=(I-\varepsilon) D+\varepsilon \cdot D^{*}-(I-\varepsilon) \omega \\
& i=\rho+\varepsilon \cdot \lambda \\
& i^{*}=\rho-(I-\varepsilon) \lambda
\end{aligned}
$$

donde,

$$
\begin{aligned}
& \omega=\left[\left(\mu-\mu^{*}\right)-n \cdot \lambda\right] \\
& \rho=-(l / n)\left[(I-\varepsilon)(D-\mu)+\varepsilon\left(D^{*}-\mu^{*}\right)\right] \\
& \varepsilon=\theta(I-f) /\left[\theta(I-f)+(I-\theta)\left(I-f^{*}\right)\right]
\end{aligned}
$$

El centro ejerce el liderazgo efectivo del sistema practicando una esterilización completa de las reservas con el fin de controlar su oferta monetaria (en cuyo caso $f^{*}=1, \theta>0$, por lo que $M^{*}=D^{*}$ ) o, alternativamente, absteniéndose de intervenir en el mercado de divisas (entonces, $\theta=1, f<1$ ). Este comportamiento asimétrico implica: ${ }^{30}$

$$
\begin{aligned}
& \varepsilon=1 \\
& i=-(I / n)\left(D^{*} \cdot \mu^{*}\right)+\lambda \\
& i^{*}=-(I / n)\left(D^{*}-\mu^{*}\right)
\end{aligned}
$$

Por consiguiente, los tipos de interés del centro se ven afectados exclusivamente por las condiciones de su propio mercado monetario, de modo que las perturbaciones internacionales de cartera (producidos

\footnotetext{
31) En el caso de un sistema simétrico, $f=f^{*}<\mathrm{I}, \theta=1 / 2, \varepsilon=\theta=$ 1/2. En un sistema simétrico cada banco central tiene un objetivo interno -por ejeinplo, el tipo de interés- $y$ un objetivo externo - por ejemplo, el tipo de cambio o el nivel de reservas-. Ambos paises coordinan sus políticas monetarias, de manera que el acervo monetario del área monetaria no se vea afectado por las perturbaciones $\left\{\mu, \mu^{*}, \lambda\right\}$ que afligen a la economía.
}

por cambios en las primas de riesgo o en las expectativas de variación del tipo de cambio) se reflejan exclusivamente en los tipos de interés de la periferia, que carga de manera exclusiva con los costos del proceso de ajuste. La esencial asimetría de este mecanismo de coordinación monetaria crea dificultades insuperables para garantizar la supervivencia del acuerdo a largo plazo.

Hasta ahora hemos supuesto que el motivo central de la periferia para utilizar un ancla cambiaria residía en su intento de "importar credibilidad" desde el centro. Exactamente el mismo resultado se alcanza si se caracteriza directamente al centro como el emisor de la moneda de reserva. Pero el comportamiento de la periferia podría deberse a la existencia de restricciones estrictas para financiar un déficit de balanza de pagos. Para ilustrar este caso supongamos a continuación que --por ejemplo como consecuencia de perturbaciones reales que afectan a la periferia- existe una distribución sesgada (asimétrica) de las perturbaciones de cartera que desplazan las preferencias en favor de la divisa del centro: si reinterpretamos $\mu$ y $\mu^{*}$ como perturbaciones reales (por ejemplo una perturbación fiscal) que afectan a la demanda de dinero, entonces $\lambda$ $=\lambda(\mu)$, y $\lambda^{\prime}(\mu)=\partial \lambda \_/ \partial \mu>0$. El efecto de un aumento de $\mu$ sobre las reservas de la periferia es igual a

$$
\partial R / \partial \mu=[\varepsilon / L \cdot f)] \cdot[1-n(\partial \lambda \partial \mu)]
$$

La expansión de la demanda generaría un aumento incipiente del tipo de interés interno que estimula la entrada de capitales y hace aumentar las reservas (esto explica el primer término de la expresión anterior). Al mismo tiempo el aumento inducido de $\lambda$ eleva el rendimiento de los activos denominados en la moneda del centro, provocando la salida de capitales y la reducción de las reservas (esto explica el segundo término de la expresión 6). Si el segundo efecto domina al primero, de modo que $\partial N \partial \mu>1 / n$, el efecto neto será una reducción de las reservas. En ese caso, dado que $\varepsilon(I-f)=\theta /\left[\theta(I-f)+(I-\theta)\left(I-f^{*}\right)\right]$, la ecuación [6] también muestra que para una regla de intervención dada, $\theta>0$, el intento por parte de la periferia de esterilizar la reducción de las reservas (mediante un aumento del crédito interno), haría aumentar el ritmo al que éstas disminuyen. La reducción de las reservas se minimiza fijando que $f=0$. Por lo tanto, en presencia de perturbaciones asimétricas suficientemente intensas y de reservas limitadas la periferia tiende a comportarse como un seguidor natural. Este comportamiento no garantiza, sin embargo, la convergencia de 
los ciclos económicos del centro y la periferia, lo que introduce complicaciones adicionales para la sostenibilidad de este modelo. ${ }^{3 !}$

Un problema adicional, de gran importancia, atañe a las dificultades para distribuir equitativamente los costos de ajuste de una devaluación selectiva en la periferia cuando existen asimetrías o diferencias estructurales entre los países periféricos. El problema se plantearía, por ejemplo, si se produce una perturbación real en el centro, grande pero no lo suficientemente intensa como para justificar una devaluación en el conjunto de la periferia. Sin mecanismos fiscales que permitan compensar a los países que soportan mayores costos de ajuste sería muy difícil coordinar la devaluación, con lo cual se inhibiría el necesario ajuste del tipo de cambio y se crearían las condiciones para una apreciación real que haría insostenible el acuerdo cooperativo, 32

\section{VII}

\section{Una propuesta de coordinación macroeconómica en el Mercosur}

La clara apuesta por la estabilidad que ha orientado en los últimos años la política económica en el Mercosur ya está produciendo un cierto grado de coordinación que podría facilitar en gran medida un acuerdo sambiario a partir de las realidades actuales. De hecho, el principal problema para la coordinación macroecooómica hasta la puesta en marcha del Plan Real por parte de Brasil residía en el diferente ritmo de la estabilización macroeconómica en el bloque regional. El que en el pasado inmediato se haya tenido que recurrir al tipo de cambio como ancla nominal no puede enmascarar el hecho de que las economías del Mercosur se encuentran abocadas a profundas translormaciones estructurales que podrían verse facilitadas por un grado suficiente de flexibilidad cambiaria. Por otra parte, no cabe duda de que una fluctuación excesiva de los tipos de cambio podría perjudicar los intercambios comerciales y la inversión, debilitando así la realización del mercado interior en el Mercosur y poniendo en peligro la sostenibilidad del proceso de apertura. La implantación de un acuerdo cambiario bien articulado e internamente consistente, basado en

\footnotetext{
${ }^{31}$ Por ejemplo, la asincronía de los ciclos económicos de Hong kong (cuyo sistema monetario está basado en un mecanismo de junta monetaria, con un tipo de cambio vinculado al dólar) y de Fistados Unidos creó serias dificultades a Hong Kong en el período 1989-1992. La caída de los tipos de interés en Estados Unidos se produjo en un momento de fuerte expansión de la economía de Flong Kong. Las presiones inflacionistas y la baja de los tipos de iuterés nominales produjeron tipos de interés reales negativos. El desplazatniento de cartera resultante - de títulos de deuda a acciones y activos reales- provocó en 1993 una subida de 1 I $6 \%$ en el precio de las acciones y de $70 \%$ en el precio de los inmuebles.
}

un mecanismo de bandas de fluctuación del tipo de cambio, podría permitir una coordinación eficaz, flexible y sostenible de las políticas macroeconómicas de los países del Mercosur. ${ }^{33}$ La coordinación macroeconómica debería prevenir, simultáneamente, la posibilidad de desalineamientos prolongados del tipo de cambio real con respecto a su valor de equilibrio de largo plazo, y el recurso a la utilización arbitraria del tipo de cambio para lograr ventajas nacionales a costa del resto de los socios comerciales (practicando políticas de desinflación competitiva o devaluaciones competitivas).

Un acuerdo cambiario organizado sobre las bases que se exponen a continuación permitiría, a mi juicio, definir un sistema monetario comparativamente satisfactorio para el conjunto del área, que además podría proporcionar a Argentina la cobertura necesaria para encontrar una estrategia de salida no traumática al sistema de cuasi caja de conversión, conservando los logros ya alcanzados en materia de estabilidad de precios:

i) En primer lugar, Argentina y Brasil acordarían particjpar, en un plazo razonable, en un mecanismo de tipos de cambio e intervención, basado en el establecimiento de una banda de fluctuación alrededor de una paridad central, y se comprometerían a defenderlo

\footnotetext{
${ }^{12}$ Buiter, Corsetti y Pesenti (1995) muestran que en este escenario la pérdida global de bienestar se aliviaría parcialmente retornando a un equilibrio de Nash.

${ }^{33}$ Los problemas relativos a la flexibilización del sistema de cuasi caja de conversión que es necesario para la ptesta en marcha de este acuerdo cambiario, se examinan brevemente en la próxima sección.
} 
activamente. El tipo de cambio de cada moneda en el interior de la banda se determinaría libremente en el mercado de divisas. ${ }^{34}$ Además, podrían negociarse aumentos transitorios de la banda en situaciones extraordinarias con el fin de desalentar los ataques especulativos contra las monedas del sistema. Gradualmente se promoverían los mecanismos financieros adecuados para facilitar la intervención conjunta en los mer* cados de cambios. El acuerdo cambiario argentinobrasileño estaría abierto a la participación voluntaria de Paraguay y Uruguay y también de aquellos países del entorno regional del Mercosur que mantienen relaciones preferenciales con este bloque, como Bolivia y Chile. La participación de otros países se haría inicialmente sobre la base de una amplitud diferenciada de la bandas cambiarias, hasta que la convergencia nominal de las diferentes economías permita unificar el mecanismo de tipos de cambio.

ii) La paridad central de cada moneda, con respecto a la cual se fijan las bandas de fluctuación, se calcularía diariamente sobre la base de una cesta monetaria que tuviese en cuenta las realidades del comercio internacional del bloque regional en su conjunto, y que estaría integrada tentativamente por el dólar $(40 \%)$, el euro $(40 \%)$ y el yen $(20 \%)$. La paridad central de cada moneda con respecto a la canasta de referencia podría modificarse periódicamente con el fin de evitar desalineamientos prolongados con respecto al tipo de cambio real de equilibrio de largo plazo.

iii) Los realineamientos de paridades se realizarían previo acuerdo de un comité monetario, institución supranacional integrada en parte mayoritaria por expertos verdaderamente independientes y en parte por representantes de los bancos centrales de los países que conforman el Mercosur; este comité se ocuparía de evitar la utilización inapropiada de las devaluaciones. En el contexto de una zona objetivo, la regulación de los saldos reales mediante intervenciones intramarginales discretas (asociadas a reglas de realineamiento del tipo de cambio nominal) puede ser muy útil para

\footnotetext{
14 Para que ese compromiso cambiario sea sostenible los gobiernos deben acatar dos reglas básicas de comportamiento: i) renunciar a la defensa de un tipo de cambio claramente desalineado con respecto al tipo de equilibrio, y ii) renunciar a renlineamientos de paridades que sobrepasen la anplitud de las bandas. Teniendo en cuenta las anteriores consideraciones, la amplitud de la banda (alrededor de la paridad centraj) no debería ser inferior al $10 \%$. Por ejemplo, ea el caso del sMe la ampliación de la banda de fluctuación en agosto de 1993 (desde el $\pm 2.25 \%$ al $\pm 15 \%$ ) contribuyó en gran medida a restautar ta credibilidad del SME, poniendo fin a los ataques especulativos contra las monedas mús débiles del sistema. Véase en Williamson (1993) un examen de los principales problemas asociados al funcionamiento correcto de un sistema de zonas objetivos.
}

complementar la intervención marginal infinitesimal en defensa de la banda. Por lo tanto, el comité monetario conjunto debería retener entre sus competencias la función de propiciar intervenciones intramarginales $y$, sobre todo, de promover modificaciones en los tipos de cambio de los países miembros si llega a la conclusión de que la paridad central es inconsistente con los fundamentos macroeconómicos. Este procedimiento permitiría aumentar la credibilidad de la política cambiaria y haría disminuir la posibilidad de ataques especulativos dirigidos a corregir el desvío del tipo de cambio con respecto a su nivel de equilibrio de largo plazo. A lo largo del tiempo el acuerdo podría complementarse con un sistema de transferencias que proveyese de mecanismos de compensación para lograr una más equitativa distribución de los costos de ajuste que entrañaría una devaluación selectiva.

iv) La amplitud relativamente grande de la banda de fluctuación y la orientación del acuerdo cambiario hacia la preservación a mediano plazo del tipo de cambio real de equilibrio, priva al tipo de cambio de su función de ancla nominal. Por lo tanto, para que el mecanismo de tipos de cambio sea consistente con el control de la inflación en el ámbito del Mercosur, sería necesario, ante todo, complementar el acuerdo cambiario con criterios de convergencia nominal (especialmente en materia fiscal) que aseguren el mantenimiento de la estabilidad de precios y la disciplina presupuestaria (limitando estrictamente las operaciones de préstamo por parte de los bancos centrales a los gobiernos y la monetización de los déficit públicos). Luego, habría que realizar urgentemente las reformas estructurales necesarias para flexibilizar los mercados (especialmente en el sector servicios y algunos subsectores del mercado de trabajo) y prevenir la reintroducción de mecanismos de indización en los contratos. Si los criterios de convergencia no se cumpliesen en un plazo razonable y las reformas estructurales no se abordasen con suficiente profundidad, los países podrían caer en la tentación de recurrir a políticas de desinflación competitiva, lo que supondría el abandono del acuerdo cooperativo.

v) Las mayores dificultades para el avance de la apertura comercial de los países en vías de desarrollo en el actual escenario de globalización financiera provienen de los riesgos asociados a la apertura financiera irrestricta. En cuanto a las posibilidades de lograr una coordinación macroeconómica efectiva, no cabe duda de que la convergencia en la ejecución y la orientación de las políticas se ha visto cada vez más difjcultada por la globalización y la creciente sofisticación de los mercados financieros. Si bien la libertad de movimientos de los capitales internacionales contribu- 
ye de manera decisiva a la correcta asignación de los recursos a nivel mundial, la inestabilidad potencial de los flujos de capitales puede introducir graves distorsiones en los sistemas financieros de los países en vías de desarrollo y producir una variabilidad ex. cesiva en el tipo de cambio y en las magnitudes monetarias. Las restricciones moderadas a los movimienos de capitales pueden contribuir de forma efectiva a estabilizar el tipo de cambio en el interior de la banda.
Esto se debe a que los controles de capitales refuerzan el peso de las expectativas en la determinación del tipo de cambio, aumentando con ello la capacidad estabilizadora de la banda. ${ }^{35} \mathrm{La}$ experiencia reciente de varios países en desarrollo (como Colombia, Chile y Malasia) muestra que una apertura financiera sistemática pero gradual elimina riesgos innecesarios asociados a capitales especulativos cuya contribución al crecimiento económico resulta marginal. ${ }^{36}$

\section{VIII}

\section{Consideraciones finales}

L_a utilización de anclas nominales demasiado rígidas se traduce en una inconsistencia que termina haciéndose fatalmente patente entre el objetivo de la política Inonetaria y la evolución de las variables macroeconómicas fundamentales. Por otra parte la sustitución recurrente de un ancla nominal por otra revela que cuando un gobierno adopta un ancla nominal cuya estabi-

\footnotetext{
${ }^{35}$ En el marco de un modelo monetario simple de bandas de fluctuacion es posible mostrar de manera muy sencilla el modo en que las restricciones a los movimientos de capitales contribuyen a reforzar la estabilización del tipo de cambio dentro de la banda. El tipo de cambio en cualquier momento del tiempo depende en ese caso de las variables fundamentales (sumarizadas por la variable $x$ ) y de la tasa de variación esperada del tipo de cambio. El tipo de cambio puede interpretarse como el valor presente descontado de las realizaciones futuras de las variables fundamentales. Con perfiscta movilidad del capital obtenemos lo sigutente (utilizando la nuisma notación que en el apéndice):
}

$$
\mathrm{s}_{1}=\frac{1}{n} E_{1} \int_{1}^{\infty} x_{\tau} \cdot e^{-\left(\frac{1}{11}\right)(z-1)} d \tau
$$

donde $x=m+v$; siendo $v=-m^{*}-h\left(y-y^{*}\right)$ la expresión de la velocidad del dinero relevante para esta economía. Diferenciando esta ezuación con respecto al tiempo $(t)$ obtenemos la expresión que gobierna el comportamiento del tipo de cambio: $s=x+n \cdot E[d s / d t]$. Con imperfecta movilidad del capital la condición de arbitraje de iutereses es: $\kappa(i \cdot i)=E(d / / d t$ ), donde $0 \leq \mathrm{x} \leq \mathrm{J}$ (sin movilidad del capital $x=0$; con perfecta movilidad del capital $\kappa=1$ ). En e:te caso el tipo de cambio vendra determinado por la ecuación $s=x+(n / \kappa) \cdot E[d s / d l]$.

*a aplicación de una legislación similar a la de Chile al conjunto del Mercosur obligaría a que la captación de financiamiento internxicional (vía préstainos o emisiones de capital) contase con la auturización del banco central de cada país y se gravase con un impuesto del $4 \%$. Además debería realizarse un depósito sin remuneráıción en el banco central por un importe del $30 \%$ del préstamo olstenido durante el plazo de un affo. De manera alternativa este dipósito podría sustituirse por el pago al banco central de intereses sobre el $30 \%$ del prestamo (calculados sumando un $4 \%$ al tipo libor) dırante un año (aun cuando el plazo de financiación sea inferior). lización se encomienda a la política monetaria, la defensa de esa variable nominal no constituye en realidad el principal objetivo de la política económica. ${ }^{37}$ Sin embargo, en algunas ocasiones la permanencia de una regla monetaria deja de ser un ejemplo de la virtud del gobierno (de la consistencia temporal de las políticas) para convertirse en un ejemplo de persistencia en el error. ${ }^{38}$ Tras los indudables logros aicanzados en materia de estabilidad de precios en el seno del Mercosur, y reconociendo los servicios prestados al anclaje de las expectativas por esquemas monetarios y cambiarios necesariamente rígidos en el momento de su aplicación, parece llegada la hora de plantear una transición hacia mecanismos de coordinación más flexibles que preserven los equilibrios macroeconómicos y que al mismo tiempo favorezcan el crecimiento sostenido en el conjunto del área.

El objetivo último del acuerdo cambiario descrito en la sección anterior es la articulación de un mecanismo de coordinación que posibilite el abandono gradual por parte de las economías del mercosur del tipo de cambio como ancla nominal, con el propósito de evitar apreciaciones (depreciaciones) reales excesivas, no justificadas por las variables macroeconómicas fundamentales, que amenazan la consolidación de un

\footnotetext{
${ }^{37}$ La literatura sobre crisis de balanza de pagos y ataques especullativos está basada en esta idea. Véuse, por ejempio, Flood y Mussa (1994).

${ }^{3 *}$ Como afirma Kindleberger (1988, pp. 201 y 207), en un magnífico ensayo, "la mayor parte de las reglas necesitan excepciones en las situaciones difíciles... una actuación de pasiva conformidad con el espiritu de las reglas es parte de la responsabilidad (económica); pero la capacidad de intervenir para aplicat y adaptar las reglas a las nuevas circunstancias es la otra parte y la más activa de dicha responsabilidad".
} 
verdadero mercado interior de alcance regional. En la actualidad las principales dificultades para el ejercicio de una coordinación macroeconómica efectiva en el Mercosur las plantea Argentina, debido a los dos principales hechos estilizados que caracterizan el funcionamiento del mecanismo de cuasi caja de conversión: su incapacidad para actuar como prestamista de última instancia y el sesgo procíclico que imprime a la economía real.

En primer lugat, la capacidad de los bancos centrales para actuar como prestamistas de última instancia es vital para que la coordinación macroeconómica sobreviva a los avatares de una crisis financiera (sobre todo dada la relativa debilidad de los sistemas bancarios argentino y brasileño). Sin embargo, el funcionamiento correcto de una cuasi caja de conversión impide el ejercicio de esa función esencial de un banco central, ya que las desviaciones de la regla de convertibilidad se pagan con un rápido deterioro de la credibilidad. De hecho, en situaciones de crisis la cuasi caja de conversión tiende a convertirse en el deudor de última instancia del sistema.

En segundo lugar, la naturaleza esencialmente procíclica de este mecanismo, al tiempo que incrementa la demanda social de coordinación macroeconómica con el fin de facilitar una suavización del ciclo económico, le quita utilidad para erradicar las indeseables aceleraciones y retrocesos intermitentes que han caracterizado tradicionalmente la evolución de las economías en desarrollo. Por otra parte, la transformación del sistema monetario en Argentina podría parecer inoportuna en un momento en que empieza a vislumbrarse un horizonte de clara recuperación económica (en e] que "estamos mal pero vamos bien", parafraseando al presidente Carlos Menem), con abundancia renovada de inversiones extranjeras llamando a la puerta de las economías emergentes. ${ }^{39}$ Sin embargo hay que tener en cuenta que es muy difícil prescindir del citado mecanismo en el entorno de incertidumbre que suele acompañar las fases recesivas, porque es entonces cuando la defensa del ancla nominal se convierte en la prioridad absoluta de la política.

En cuanto a la estrategia para abandonar este mecanismo en Argentina parece razonable apostar por una transición ordenada, que preserve durante algún tiempo ciertos elementos de la actual cuasi caja de conversión con el propósito de minimizar las repercusiones del cambio de régimen sobre los mercados financieros, pero que permita al banco central actuar como prestamista de última instancia y administrar el tipo de cambio en el marco del acuerdo cambiario. El precedente de Singapur (que también parece guiar la reciente evolución de Hong Kong) podría resultar de alguna utilidad a este respecto. ${ }^{40}$

\footnotetext{
${ }^{39}$ La economía argentina alcanzó una tasa media de crecimiento real del $7.7 \%$ en el período 1991-1994, sufriendo a continuación una severa corrección deflacionaria que supuso un crecimiento negativo dei producto de $4.4 \%$ en 1995 y un aumento espectacular de la tasa de desempleo (que llegó a triplicar su promedio historico) a la que se está haciendo frente con niveles infimos de cobertura social. En 1996 se produjo una recuperación moderada (el pa crecio a tasas cercanas al $4 \%$ ) que apenas tuvo consecuencias positivas para el desempleo. Al mismo tiempo, la tasa de ahorto se sitúa en Argentina en torno al $15 \%$ del PIB, por debajo del $18 \%$ de promedio en América Latina, y muy por debajo de las tasas alcanzadas en Chile (cercanas at 28\%) o en los países del este de Asia (33\%).
}

\begin{abstract}
4) Véase, por ejemplo, Osband y Villanueva (1993) y Schwartz (1993). La autoridad monetaria de Singapur, tras abandonar el régimen de tipos de cambio fijos, fue recobrando gradualmente las competencias esenciales de un banco central (capacidad para realj. zar operaciones de mercado abierto, de esterilizar las reservas, etc.), al tiempo que ha continuado respaldando el $100 \%$ de la base mo. netaria con reservas externas.
\end{abstract}


Las ventajas relativas de la coordinación macroeconómica pueden considerarse de forma sintética a partir de un sencillo modelo de dos países. El modelo es logarítmico-lineal. Las ecuaciones correspondientes al país A observan una perfecta simetría con las del país $B$ (que se refleja en la existencia de parámetros estructurales similares). Las siguientes ecuaciones describen la estructura de la economía:

$$
\begin{array}{ll}
m-p=h y-n i & \text { [A.1] } \\
y=\delta e-\sigma i+\mu & \text { [A.2] } \\
i=i^{*} & \text { [A.3] } \\
e=s+p^{*}-p & \text { [A.4] } \\
q=\alpha p+(l-\alpha)\left(s+p^{*}\right) & \text { [A.5] }
\end{array}
$$

A continuación se definen las variables y parámetros del modelo (las variables del país B se distinguen mediante un asterisco y todas las variables, con la excepción de los tipos de interés, se representan como desviaciones porcentuales con respecto a sus niveles de equilibrio de largo plazo o, alternativamente, como tasas de variación):

$m=$ acervo monetario

$p=$ índice de precios de la producción interna

$p_{0}=$ perturbación de precios inicial

$y=$ ingreso real

$i=$ nivel del tipo de interés nominal

$s=$ tipo de cambio nominal

$z=$ tipo de cambio real

$i=$ perturbación fiscal

$q=$ índice de precios al consumidor

$i=$ elasticidad-ingreso de la demanda de dinero

$n=$ semielasticidad de la demanda de dinero con respecto al tipo de interés

$\delta=$ elasticidad de la demanda de bienes con respecto al tipo de cambio real

$\sigma=$ semielasticidad de la demanda de bienes con respecto al tipo de interés

$\alpha=$ peso de la producción interna en el índice de precios al consumidor.

La ecuación [A.1] representa el equilibrio en el mercado monetario. La ecuación [A.2] es la condición de equilibrio en el mercado de bienes y servicios. La tcuación [A.3] representa la condición de arbitraje de intereses, bajo el supuesto de expectativas estáticas. La scuación [A.4] define el (logaritmo del) típo de cambio real. La ecuación [A.5] muestra el cambio porcentual del índice de precios al consumidor en función de las tasas de variación correspondientes del precio de los productos importados y del precio de la producción interna. La ecuación [A.6] introduce el supuesto de que la tasa de variación de los precios de los bienes fabricados en cada país está dada ( $y$ es, por razones de sjmetría, idéntica en ambos países).

Las ecuaciones estructurales [A.1] a [A.6] de ambos países pueden resolverse para proporcionar ecuaciones en forma reducida de $\{y, q\}$ en función de las dos variables de política $\left\{m, m^{*}\right\}$ y de la perturbación de precios simétrica y exógena $\left(p_{0}\right)$. A través de una sencilla manipulación de términos obtenemos las siguientes expresiones:

$$
\begin{aligned}
& y=a m \cdot b m^{*} \cdot(a-b) p_{0} \\
& y^{*}=a m^{*}-b m-(a-b) p_{0} \\
& s=(1 / 2 \delta h)\left(m \cdot m^{*}\right) \\
& q=p_{0}+\gamma\left(m-m^{*}\right) \\
& q^{*}=p_{0}-\gamma\left(m-m^{*}\right)
\end{aligned}
$$

donde,

$$
\begin{aligned}
& \mathrm{b}=\frac{n}{2 h(n+h \sigma)}>0, \quad \mathrm{a}=\frac{n+2 h \sigma}{2 h(n+h \sigma)}>0, \\
& \mathrm{a}-\mathrm{b}=\frac{\sigma}{n+h \sigma}>0, \quad \gamma=\frac{l \cdot \alpha}{2 h \sigma)}>0
\end{aligned}
$$

Cada gobierno intenta minimizar la desviación de la producción y de la inflación con respecto a los niveles deseados de equilibrio (normalizados a cero). La existencia de rigideces fiscales implica $\mu=0$ en la ecuación [A.2], de modo que la política monetaria es el único instrumento de política. La funnción de pérdidas en el caso del país A es:

$$
\pi=y^{2}+\beta q^{2}
$$

donde el parámetro $\beta$ refleja la importancia relativa que el gobierno concede a $q^{41}$

Consideremos en primer lugar el equilibrio no cooperativo de Cournot-Nash. La función de reacción en el caso del país A es,

\footnotetext{
4' La función de pérdidas se supone simétrica para aumentos y disminuciones de las variables $\{y, q\}$, de manera que cambios en cualquier dirección de estas variables causan desutilidad. Con el fin de simplificar fa exposición, consideraremos que las funciones de pérdidas de ambos países son perfectamente simétricas (de modo que $\beta=\beta^{\circ}$ ) y estables a lo largo del tiempo.
} 


$$
\begin{aligned}
& m=A m^{*}+B p_{0} \\
& A=\frac{a b+\beta \gamma^{2}}{a^{2}+\beta \gamma^{2}}<1, \text { dado que } a>b,
\end{aligned}
$$

donde,

$$
B=\frac{A(a-b)-\beta \gamma}{a^{2}+\beta \gamma^{2}}>0, \text { si } \frac{a(a-b)}{\gamma} \beta
$$

De igual manera, por simetría, se obtiene la función de reacción del país $\mathrm{B}$ :

$$
m^{*}=A m+B p_{0}
$$

El equilibrio de Cournot-Nash se alcanza en el punto de intersección de las funciones de reacción y por lo tanto se verifica que,

$$
m=m^{*}
$$

Sustituyendo la expresión anterior en la función de reacción del país A (ecuación [A.13]) obtenemos la condición de equilibrio de Cournot-Nash en función de los parámetros estructurales del modelo y de $p_{0}$ :

$$
m=m^{*}=\frac{a(a-b)-\beta \gamma}{a(a-b)} p_{0}
$$

Por otra parte, sustituyendo la ecuación [A.15] en las ecuaciones [A.7] a [A.1 I], y teniendo en cuenta la ecuación [A. 16], llegamos a la conclusión de que, en la situación de equilibrio, se verifica que:

$$
\begin{aligned}
& s=0 \\
& q=q^{*}=p_{0} \\
& y=y^{*}=(-\beta \gamma / a) p_{0}<0
\end{aligned}
$$

Por consiguiente, en respuesta a la perturbación de precios inicial $\left(p_{0}\right)$ la política monetaria de ambos países resulta excesivamente contractiva, al aumentar los tipos de interés y reducir la producción por debajo del nivel deseado en cada país. Cabe señalar que el grado de contracción monetaria de este equilibrio no cooperativo depende del peso relativo de la inflación en la función de pérdidas. Si éste es relativamente elevado (de modo que $a(a-b) / \gamma<\beta$, y en consecuencia, $m=m^{*}<s=0<p_{0}$ ), la reducción de los niveles de producción será también mayor. Alternativamente, cuando $a(a-b) / \gamma \beta$, se verifica que $p_{0}>m=m>s=0$.

En el equilibrio cooperativo el comité monetario conjunto resuelve el siguiente programa:

Min. $\left(\pi+\pi^{*}\right)=\left(y^{2}+\beta q^{2}\right)+\left(y^{* 2}+\beta q^{* 2}\right)$,

$$
[m, m *]
$$

sujeto a:

$$
\begin{aligned}
& m=m^{*} \\
& q=q^{*}=p_{0} \\
& y=y^{*}=(a-b)\left(m-p_{0}\right)
\end{aligned}
$$

La solución que se alcanza es la siguiente:

$$
\begin{aligned}
& m=m^{*}=p_{0} \\
& y=y^{*}=0
\end{aligned}
$$

\section{Bibliografía}

Bayoumi. T. y B. Eichengreen (1994): One money or many? Analyzing the prospects for monetary unification in various parts of the world, Princeton Stadies in International Finance, $\mathrm{N}^{*} 76$, Princeton, Princeton University Press.

Buiter, W. H. (1995); Macroeconomic Policy During a Trunsition to Monetary Union, Discussion Paper, $\mathrm{N}^{*} 26 \mathrm{~J}$, Londres, Center for Economic Performance.

Buiter, W. H., G. Corsetti y P. Pesenti (1995): A Center-Periphery Model of Monetary Coordination and Exchange Rate Crisis. Discussion Paper $N^{\circ} 246$, Londres, Center for Economic Performance.

Connolly, M. B. (1995): The uses of a currency board: Argentina: April 1. 199I to the present, Note Economiche, vol. 24, ND 3, Siena, Banca Monte dei Paschi di Siena.

Cooper, R.N. (1976): Worldwide regional integration: is there an optimal size of the integrated area?, Economic Policy in an Interdependent World. Essays in World Economics, Cambridge, Massachusetts, MIT Press.
Crockett, A. (1989): The role of international institutions in surveillance and policy coordination, R. C. Bryant y otros (eds.), Macroeconsmic Policies in an Inserdependens World. Wastington, D.C., Fondo Monetario Internacional (FMI).

Cursie, D. (1993): Istemational cooperation in monetary policy: Has it a future?, The Economic Journal, vol. 103, No 416, Londres, The Royal Ecomomic Society.

Feldsteit, M. (1992): The case against EMU, The Economist, vol. 323, N $\mathrm{N}^{\circ} 7763$, Londres, 13 de junio.

Ferter, A, y R. Lavagna (J992): Mercosur y coordinación de políticas económicas, M. Bekerman (ed.), Mercosur. La oportunidad y el desafio, Buenos Aires, Editorial Legasa.

Flood, R. y M. Mussa (1994): Issues concerning nominal anchors for monetary policy, NBER Working Paper, $\mathrm{N}^{\circ} 4850$, Cambridge, Massachusetts, National Buteal of Economic Research, luc. (NBER).

Giovannini, A. (1988): How do fixed-exchange-rate regimes work: The evidence from the gold standard, Bretton Woods and the 
EMS, NBER Working Paper, $\mathrm{N}^{\circ}$ 2766, Cambtidge, Massachusetts, NBER.

Hamada, K. ([976): A strategic analysis of monetary interdependence, Journal of Political Economy, vol. 84, No 4, Chicago, University of Chicago Press, agosto.

(1979): Macroeconomic strategy and coordination under alternative exhange rates, R. Dornbusch y J. A. Frenkel (eds.), International Economic Policy: Theory and Evidence, Baltimore, Johns Hopkins University Press.

Heyman D. (1992): Notes on very high inflation and stabilization, A. Vercelli y N. Dimitri (eds.), Macroeconomics. A Survey of Research Strategies, Oxford, Reino Unido, Oxford University Press.

Heymann, D. y F. Navajas (1992): Coordinacion de polticus macroeconómicas. Aspectos conceptuales vinculados con el Mercesur, Documento de trabajo, $\mathrm{N}^{\circ} 45$, LC/BUE/L.127, Buenos Aires, Oficina de la CEPAL en Buenos Aires.

Judy, R. W. (1995): Currency Bourds: An ldea Whose Time has Come?, Washington, D.C., Centro Internacional para la Empresa Privada (CIPE).

Kenen, P.B. (1969): The theory of optimum currency areas: An eclectic view, R.A. Mundell y A.K. Swoboda (eds.), Monefary Problems of the International Ecronomy. Chicago, Illinois, University of Chicago Press.

Kindleberger, Ch.P. (1988): Economic Responsability, Ch.P. Kindleberger (ed.), The International Ecomomic Order. Essays on Financial Crisis and International Pablic Goods, Cambridge, Massaclusetts, MiT Press.

Krugman, P. R. (1988): Target zones and exchange rate dynamics, NBER Working Paper, No 2481, Cambridge, Massachusetts, NGER, enero.

(1990); Policy problems of a monetary union, P.De Grauwe y L. Papademos (eds.): The European Monetary System in the 1990 's. Londres, Center for Earopean Policy Studies/Banco de Grecia.

McKibbin, W. J. y J. D. Sachs (1991): Global Linkages. Macroeconomic Interdependence and Cooperation in the World Economy, Washington, D.C.The Brookings Insticution.

McKimnon, R. I. (1963): Optimum eutrency areus. The American Economic Review, vol. $53, \mathrm{~N}^{\circ} 4$, Los Angeles, California, American Economic Association.

Mundell, R. A. (1961): A theory of optimum currency areas. The American Economic Review, vol. 5I, N" 4, Los Angeles, California, America Economic Association.

Osband, K. y D. Villanueva (1993): Independent Currency
Authorities. An Analytic Primer, IMF staff papers, vol. 40, Na 1 , Washington, D.C., FMI.

Oudiz, G. y J. Sachs (1986): Intemational policy coordination in dynamic models, W. Buiter y R. Marston (eds.); Intemational Economic Policy Coordination. Cambridge, Reino Unido, Cambridge University Press.

Poole, W. (1970): Optimal choice of monetary policy instruments in a simple stochastic macro model, Quarterly Journat of Economics, vol. 84, $\mathrm{N}^{\circ} 2$, Cambridge, Massachusetts, Harvard University, mayo.

Quirk, P. J. (1996): Exchange Rate Regimes as Inflation Anchors, Washington, D.C., George Washington University, Institute of Brazilian Business and Public Management Issues.

Rodríguez Prada, G. (1994): La programación económico-financieta en un sistema de CURRENCY BOARO, Textos de economía, vol. 5, N" 1, Florianopolts, Brasil, Universidade Federal de Santa Catarina.

(1995): La economía de los CURRENCY BOARDS: pasado y presente, Economirtas, № 63 , Madrid, Colegio de Economistas de Madrid.

(1996): El tipo de cambio como ancla nominal, CURRENCY BOARDS y dolarización. G. Rodríguez Prada (ed.), La macro economía de los mercados emergentes, Alcalá de Henares, Ediciones de la Universidad de Alcalá.

Sachs, J. y A. A. Zini (1996): Brazilian inflation and the Plano Real, The World Economy, vol. 19, № 1, Oxford, Reino Unido.

Schwartz, A. J. (1993): Currency boards: their past, present and possible future role, Carnegie Rochester Papers on Public Policy, vol. 39, Amsterdam, North-Holland Publishing Company.

Williamson, J. (1993): Exchange rate management, The Economic Journal, vol. 103, N446. Cambridge, Reino Unido, The Royal Economic Society.

(1995): What Role for Currency Boards?, Washington, D.C., Institute for International Economics (IIE).

Williunson, J. y M. H. Milter (1987): Targets and Indicators: A Blueprint for the Interuational Coordination of Economic Policy. Policy Analywis in International Economics, N" 22 , Washington, D. C., IIE.

Whitman, M. N. (1977): Sustaining the International Economic System, Essays in International Finance, $N^{\circ} 121$, Princeton, Princeton University.

Zarazaga, C. E. (1995): Can currency boards prevent devaluations and funancial meltdowns? The Southwest Economy, $\mathrm{N}^{*} 4$, Dallas, Federal Reserve Bank of Daltas. 